\title{
OSCILLATORY PROPERTIES OF CERTAIN SYSTEM OF NON-LINEAR ORDINARY DIFFERENTIAL EQUATIONS
}

\author{
ZDENĚK OPLUŠTIL
}

Received 06 September, 2017

Abstract. We consider the two-dimensional system of non-linear differential equations

$$
u^{\prime}=g(t)|v|^{\frac{1}{\alpha}} \operatorname{sgn} v, \quad v^{\prime}=-p(t)|u|^{\alpha} \operatorname{sgn} u,
$$

where $\alpha>0, g:[0,+\infty[\rightarrow[0,+\infty[$, and $p:[0,+\infty[\rightarrow \mathbb{R}$ are locally integrable functions. In the case $\int^{+\infty} g(s) d s=+\infty$, the considered system has been widely studied in particular cases such linear systems as well as second order linear and half-linear differential equations. However, the case $\int^{+\infty} g(s) d s<+\infty$ has not been studied in detail in the existing literature. Moreover, we allow that the coefficient $g$ can have zero points in any neighbourhood of infinity and consequently, considered system can not be rewritten as the second order linear or half-linear differential equation in this case. In the paper, new oscillation criteria are established in the case $\int^{+\infty} g(s) d s<+\infty$ and without restricted assumption function p preserves its sign (which is usually considered).

2000 Mathematics Subject Classification: 34C10

Keywords: two dimensional system of non-linear differential equations, oscillatory properties

\section{INTRODUCTION}

On the half-line $\mathbb{R}_{+}=[0,+\infty[$, we consider the two-dimensional system of nonlinear ordinary differential equations

$$
\begin{aligned}
& u^{\prime}=g(t)|v|^{\frac{1}{\alpha}} \operatorname{sgn} v, \\
& v^{\prime}=-p(t)|u|^{\alpha} \operatorname{sgn} u,
\end{aligned}
$$

where $\alpha>0$ and $p, g: \mathbb{R}_{+} \rightarrow \mathbb{R}$ are locally Lebesgue integrable functions.

Under a solution of system (1.1) on the interval $J \subseteq[0,+\infty[$ we understand a vector function $(u, v)$, where functions $u, v: J \rightarrow \mathbb{R}$ are absolutely continuous on every compact interval contained in $J$ and satisfy equalities (1.1) almost everywhere in $J$.

The published results were supported by Brno University of Technology, specific research plan no. FSI-S-17-4464 "Modern mathematical methods in modelling problems of technical and natural sciences". 
It is known (see [7]) that all non-extendable solutions of system (1.1) are defined on the whole interval $[0,+\infty$. Therefore, when we are speaking about a solution of system (1.1), we assume without loss of generality that it is defined on $[0,+\infty[$.

Definition 1. A solution $(u, v)$ of system (1.1) is called non-trivial if $u \not \equiv 0$ on any neighborhood of $+\infty$. We say that a non-trivial solution $(u, v)$ of system (1.1) is oscillatory if the function $u$ has a sequence of zeros tending to infinity, and nonoscillatory otherwise.

In [7, Theorem 1.1], it is proved that a certain analogue of Sturm's theorem holds for system (1.1), if the additional assumption

$$
g(t) \geq 0 \quad \text { for a.e. } t \geq 0
$$

is satisfied. Especially, under assumption (1.2), if system (1.1) has an oscillatory solution, then any other its non-trivial solution is also oscillatory. Moreover, if $(u, v)$ is an oscillatory solution to system (1.1) then also the function $v$ oscillates.

On the contrary, if $g \equiv 0$ on some neighborhood of $+\infty$, then all non-trivial solutions of system (1.1) are non-oscillatory. Consequently, it is natural to assume that inequality (1.2) is satisfied and

$$
\text { meas }\{\tau \geq t: g(\tau)>0\}>0 \text { for } t \geq 0 .
$$

Definition 2. We say that system (1.1) is oscillatory if all its non-trivial solutions are oscillatory.

It is clear that the half-linear equation

$$
\left(r(t)\left|u^{\prime}\right|^{q-1} \operatorname{sgn} u^{\prime}\right)^{\prime}+p(t)|u|^{q-1} \operatorname{sgn} u=0
$$

is a particular case of system (1.1). Indeed, this equation is usually studied under

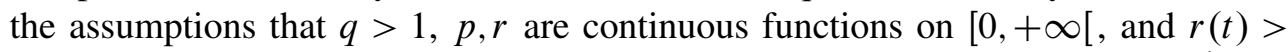
0 for $t \geq a$. If the function $u$ is a solution of equation (1.4) (i.e., $u \in C^{1}$ and $\left.r\left|u^{\prime}\right|^{q-1} \operatorname{sgn} u^{\prime} \in C^{1}\right)$, then the vector function $\left(u, r\left|u^{\prime}\right|^{q-1} \operatorname{sgn} u^{\prime}\right)$ is a solution of system (1.1) with $g(t):=r^{\frac{1}{1-q}}(t)$ and $\alpha:=q-1$.

In the case $\int_{0}^{+\infty} r^{\frac{1}{1-q}}(s) d s=+\infty$ (i.e. $\int_{0}^{+\infty} g(s) d s=+\infty$ in (1.1)), there are many interesting results in the existing literature (see, e.g., [2, 3, 5, 6, 8]). However, in the case $\int_{0}^{+\infty} r^{\frac{1}{1-q}}(s) d s<+\infty$ (i.e. $\int_{0}^{+\infty} g(s) d s<+\infty$ in (1.1)), as far as we know, only a few results are known. Namely, some Hille and Nehari type oscillations criteria are presented in the papers [4,9], where together with $\int_{0}^{+\infty} r^{\frac{1}{1-q}}(s) d s<+\infty$ is assumed that

$$
p(t) \geq 0 \quad \text { for } t \geq 0 .
$$

In addition, the coefficient $g$ can have zero points in any neighbourhood of infinity and consequently, considered system can not be rewritten as the half-linear differential equation (1.4) in generall. 
In this paper we "remove" the assumption (1.5) and we obtain oscillation criteria, which complement and generalize above mentioned results. Therefore, throughout the paper, we assume that the coefficient $g$ is integrable on $[0,+\infty[$, i.e.,

$$
\int_{0}^{+\infty} g(s) d s<+\infty
$$

Let

$$
f(t):=\int_{t}^{+\infty} g(t) d s \quad \text { for } t \geq 0 .
$$

In view of assumptions (1.2), (1.3), and (1.6), we have

$$
\lim _{t \rightarrow+\infty} f(t)=0
$$

and

$$
f(t)>0 \quad \text { for } t \geq 0 .
$$

For any $\lambda>\alpha$, we put

$$
c_{\alpha}(t ; \lambda):=(\lambda-\alpha) f^{\lambda-\alpha}(t) \int_{0}^{t} \frac{g(s)}{f^{\lambda-\alpha+1}(s)}\left(\int_{0}^{s} f^{\lambda}(\xi) p(\xi) d \xi\right) d s \quad \text { for } t \geq 0 .
$$

The following statement was established in [1].

Theorem 1 ([1, Corollary 2.11 (with $v=1-\alpha+\lambda)]$ ). Let conditions (1.2), (1.3), and (1.6) hold, $\lambda>\alpha$, and either

$$
\lim _{t \rightarrow+\infty} c_{\alpha}(t ; \lambda)=+\infty
$$

or

$$
-\infty<\liminf _{t \rightarrow+\infty} c_{\alpha}(t ; \lambda)<\limsup _{t \rightarrow+\infty} c_{\alpha}(t ; \lambda) .
$$

Then system (1.1) is oscillatory.

It is obvious that two cases are not covered by Theorem 1, namely, the function $c_{\alpha}(\cdot ; \lambda)$ has a finite limit and $\liminf _{t \rightarrow+\infty} c_{\alpha}(t ; \lambda)=-\infty$. We are interested in the first case when there exists finite limit of the function $c_{\alpha}(\cdot ; \lambda)$, i.e., if

$$
\lim _{t \rightarrow+\infty} c_{\alpha}(t ; \lambda)=: c_{\alpha}^{*}(\lambda) \in \mathbb{R} .
$$

\section{MAIN RESULTS}

This section contains formulations of all the results of he paper. Their proofs are presented in detail in Section 3.

Theorem 2. Let $\lambda>\alpha$ and (1.10) hold. Let, moreover, the inequality

$$
\limsup _{t \rightarrow+\infty} \frac{-1}{f^{\lambda-\alpha}(t) \ln f(t)}\left(c_{\alpha}^{*}(\lambda)-c_{\alpha}(t ; \lambda)\right)>\left(\frac{\alpha}{1+\alpha}\right)^{1+\alpha}
$$

be satisfied. Then system (1.1) is oscillatory. 
As we presented above, known oscillation criteria for the equation (1.4) (with integrable function $\left.r^{\frac{1}{1-q}}(t)\right)$ are established in [4,9] under sign assumption on its coefficient $p(t)$. We show an example of the system (1.1), where we can use the oscillatory critearia from the Theorem 2 , but we can not apply results from above mention papers.

Example 1. Consider the system (1.1), where $\alpha=2$

$$
g(t):=\frac{1}{(t+1)^{2}}, \quad \text { and } \quad p(t):=(t+1)^{4} \cos t \quad \text { for } t \geq 0 .
$$

Obviously, the function $p$ and its integral

$$
\begin{aligned}
\int_{0}^{t} p(s) d s & =\left(t^{4}+4 t^{3}-6 t^{2}-20 t+13\right) \sin t \\
& +\left(4 t^{3}+12 t^{2}-12 t-20\right) \cos t+20 \quad \text { for } t \geq 0
\end{aligned}
$$

change their signs in any neighbourhood of $+\infty$. Therefore neither of results stated in the papers $[4,9]$ can be used.

On the other hand, from (1.9) we obtain

$$
\begin{aligned}
c_{2}(t ; 4) & =\frac{2}{(1+t)^{2}} \int_{0}^{t}(1+s)\left(\int_{0}^{s} \cos \xi d \xi\right) d s \\
& =\frac{2}{(1+t)^{2}}(1+\sin t-t \cos t-\cos t) \quad \text { for } t \geq 0 .
\end{aligned}
$$

Hence, the function $c_{2}(\cdot, 4)$ has the finite limit

$$
c_{\alpha}^{*}(4)=\lim _{t \rightarrow+\infty} c_{2}(t ; 4)=0,
$$

i.e., (1.10) holds. Moreover,

$$
\limsup _{t \rightarrow+\infty} \frac{(t+1)^{2}}{\ln (t+1)}\left(c_{\alpha}^{*}(4)-c_{2}(t ; 4)\right)=\limsup _{t \rightarrow+\infty} \frac{2(\cos t+t \cos t-\sin t-1)}{\ln (t+1)}=+\infty .
$$

Consequently, condition (2.1) is fulfilled with $\alpha=2$ and according to Theorem 2, system (1.1) is oscillatory.

Introduce the following notations. For any $\lambda \in] \alpha,+\infty[$ and $\mu \in[0, \alpha[$, we put

$$
\begin{aligned}
& Q(t ; \alpha, \lambda):=\frac{1}{f^{\lambda-\alpha}(t)}\left(c_{\alpha}^{*}(\lambda)-\int_{0}^{t} p(s) f^{\lambda}(s) d s\right) \quad \text { for } t \geq 0, \\
& H(t ; \alpha, \mu):=f^{\alpha-\mu}(t) \int_{0}^{t} p(s) f^{\mu}(s) d s \quad \text { for } t \geq 0,
\end{aligned}
$$


where the number $c_{\alpha}^{*}(\lambda)$ is given by (1.10). Moreover, let denote

$$
\begin{array}{lll}
Q_{*}(\alpha, \lambda):=\liminf _{t \rightarrow+\infty} Q(t ; \alpha, \lambda), & H_{*}(\alpha, \mu):=\liminf _{t \rightarrow+\infty} H(t ; \alpha, \mu), \\
Q^{*}(\alpha, \lambda):=\limsup _{t \rightarrow+\infty} Q(t ; \alpha, \lambda), & H^{*}(\alpha, \mu):=\limsup _{t \rightarrow+\infty} H(t ; \alpha, \mu) .
\end{array}
$$

Theorem 2 yields the following statements.

Corollary 1. Let $\lambda>\alpha$, (1.10) hold, and $Q_{*}(\alpha, \lambda)>-\infty$. Let, moreover,

$$
\limsup _{t \rightarrow+\infty} \frac{-1}{\ln f(t)} \int_{0}^{t} f^{\alpha}(s) p(s) d s>\left(\frac{\alpha}{\alpha+1}\right)^{\alpha+1}
$$

Then system (1.1) is oscillatory.

Corollary 2. Let $\mu \in[0, \alpha[, \lambda \in] \alpha,+\infty[$, and (1.10) hold. Let, moreover,

$$
\liminf _{t \rightarrow+\infty}(Q(t ; \alpha, \lambda)+H(t ; \alpha, \mu))>\frac{(\lambda-\mu)}{(\lambda-\alpha)(\alpha-\mu)}\left(\frac{\alpha}{1+\alpha}\right)^{1+\alpha} .
$$

Then system (1.1) is oscillatory.

Corollary 3. Let $\mu \in[0, \alpha[, \lambda \in] \alpha,+\infty[$, and (1.10) hold. Let, moreover, either

$$
Q_{*}(\alpha, \lambda)>\frac{1}{\lambda-\alpha}\left(\frac{\alpha}{1+\alpha}\right)^{1+\alpha},
$$

or

$$
H_{*}(\alpha, \mu)>\frac{1}{\alpha-\mu}\left(\frac{\alpha}{1+\alpha}\right)^{1+\alpha} .
$$

Then system (1.1) is oscillatory.

Remark 1. Corollary 3 generalizes result presented in [4]. Indeed, in [4, Theorem 3.1] is established condition (2.7) with $\lambda=\alpha+1$, but there is an additional assumption $p(t) \geq 0$ for $t \geq a$. We proved oscillation criteria without this additional restrictions, so they can be apply, even if the function $p(t)$ changes its sign (see the following example).

Example 2. Consider the system (1.1), where $\alpha=2$,

$g(t):=\frac{1}{(t+1)^{2}}$, and $p(t):=(t+1)\left(\cos \left(\ln (t+1)+\sin \left(\ln (t+1)+\frac{4}{3}\right) \quad\right.\right.$ for $t \geq 0$.

Obviously, the function $p$ changes its sign in any neighbourhood of $+\infty$, i.e. we can not use the criteria presented in [4]. 
On the other hand from (1.9) we obtain

$$
\begin{aligned}
c_{2}(t ; 3) & =\frac{1}{(1+t)} \int_{0}^{t}\left(\int_{0}^{s} \frac{\cos \left(\ln (\xi+1)+\sin \left(\ln (\xi+1)+\frac{4}{3}\right.\right.}{(\xi+1)^{2}} d \xi\right) d s \\
& =\frac{7 t-4 \ln (1+t)-3 \sin (\ln (1+t))}{3(1+t)} \quad \text { for } t \geq 0 .
\end{aligned}
$$

Therefore, the function $c_{2}(\cdot, 3)$ has the finite limit

$$
c_{2}^{*}(3)=\lim _{t \rightarrow+\infty} c_{2}(t ; 3)=\frac{7}{3},
$$

i.e., (1.10) holds. In view of (2.2) we have

$$
\begin{aligned}
Q(t ; 2,3) & =(t+1)\left(\frac{7}{3}-\int_{0}^{t} \frac{\cos \left(\ln (1+s)+\sin \left(\ln (1+s)+\frac{4}{3}\right.\right.}{(1+s)^{2}} d s\right) \\
& =\cos (\ln (t+1))+\frac{4}{3} \quad \text { for } t \geq 0
\end{aligned}
$$

Hence

$$
Q_{*}(\alpha, \lambda)=\liminf _{t \rightarrow+\infty} Q(t ; 2,3)=\frac{1}{3}>\frac{8}{27}=Q_{*}(\alpha, \lambda)>\frac{1}{\lambda-\alpha}\left(\frac{\alpha}{1+\alpha}\right)^{1+\alpha}
$$

with $\alpha=2$ and $\lambda=3$. Consequently, condition (2.7) is satisfied and according to Corollary 3 , system (1.1) is oscillatory.

Theorem 3. Let $\mu \in[0, \alpha[, \lambda \in] \alpha,+\infty[$, and (1.10) hold. Let, moreover,

$$
\limsup _{t \rightarrow+\infty}(Q(t ; \alpha, \lambda)+H(t ; \alpha, \mu))>\frac{1}{\lambda-\alpha}\left(\frac{\lambda}{1+\alpha}\right)^{1+\alpha}+\frac{1}{\alpha-\mu}\left(\frac{\mu}{1+\alpha}\right)^{1+\alpha} \text {. }
$$

Then system (1.1) is oscillatory.

Remark 2. Let we notice that if $p \geq 0$ for $t \geq 0$ then conditions $Q^{*}(\alpha, \lambda)>1$ or $H^{*}(\alpha, \mu)>1$ guarantees that (2.9) with $\lambda=\alpha+1$ and $\mu=0$ is satisfied. Consequently, one can see Theorem 3 generalizes criteria established in [4, Theorem 3.5].

Now we provide two statements complementing Corollary 3 in a certain sense.

Theorem 4. Let $\mu \in[0, \alpha[, \lambda \in] \alpha,+\infty[$, and (1.10) hold. Let, moreover, the inequalities

$$
\frac{\alpha}{\lambda-\alpha}\left(\gamma-\gamma^{\frac{1+\alpha}{\alpha}}\right) \leq Q_{*}(\alpha, \lambda) \leq \frac{1}{\lambda-\alpha}\left(\frac{\alpha}{\alpha+1}\right)^{\alpha+1}
$$


and

$$
H^{*}(\alpha, \mu)>\frac{1}{\alpha-\mu}\left(\frac{\mu}{1+\alpha}\right)^{1+\alpha}+\gamma-A(\alpha, \lambda)
$$

be satisfied, where

$$
\gamma:=\left(\frac{\lambda}{1+\alpha}\right)^{\alpha}
$$

and $A(\alpha, \lambda)$ is the smallest root of the equation

$$
\alpha|x-\gamma|^{\frac{1+\alpha}{\alpha}}+\alpha x+(\lambda-\alpha) Q_{*}(\alpha, \lambda)-\alpha \gamma=0 .
$$

Then system (1.1) is oscillatory.

Theorem 5. Let $\mu \in[0, \alpha[, \lambda \in] \alpha,+\infty[$, and (1.10) hold. Let, moreover, the inequalities

$$
\left(\frac{\mu}{1+\alpha}\right)^{\alpha} \frac{\alpha(1+\alpha-\mu)}{(\alpha-\mu)(1+\alpha)} \leq H_{*}(\alpha, \mu) \leq \frac{1}{\alpha-\mu}\left(\frac{\alpha}{1+\alpha}\right)^{1+\alpha}
$$

and

$$
Q^{*}(\alpha, \lambda)>B(\alpha, \mu)+\frac{1}{\lambda-\alpha}\left(\frac{\lambda}{1+\alpha}\right)^{1+\alpha}
$$

be satisfied, where $B(\alpha, \mu)$ is the greatest root of the equation

$$
\alpha|x|^{\frac{1+\alpha}{\alpha}}+\alpha x+(\alpha-\mu) H_{*}(\alpha, \mu)=0 .
$$

Then system (1.1) is oscillatory.

Finally, we present an assertion, when both conditions (2.10) and (2.14) are fulfilled. In this case, we can obtain sharper results than those in Theorems 4 and 5.

Theorem 6. Let $\mu \in[0, \alpha[, \lambda \in] \alpha,+\infty[$, and (1.10) hold. Let, moreover, conditions (2.10) and (2.14) be satisfied and

$$
\begin{gathered}
\limsup _{t \rightarrow+\infty}(Q(t ; \alpha, \lambda)+H(t ; \alpha, \mu))>B(\alpha, \mu)-A(\alpha, \lambda) \\
+Q_{*}(\alpha, \lambda)+H_{*}(\alpha, \mu)+\gamma,
\end{gathered}
$$

where the number $\gamma$ is defined by (2.12), $A(\alpha, \lambda)$ is the smallest root of equation (2.13), and $B(\alpha, \mu)$ is the greatest root of equation (2.16). Then system (1.1) is oscillatory.

\section{PROOFS OF MAIN RESULTS}

We first formulate auxiliary lemmas, which we need to prove main statements. 
Lemma 1 ([1, Lemma 3.1]). Let $\alpha>0$ and $\omega \geq 0$. Then the inequality

$$
\omega|x|-\alpha|x|^{\frac{1+\alpha}{\alpha}} \leq\left(\frac{\omega}{1+\alpha}\right)^{1+\alpha}
$$

is satisfied for all $x \in \mathbb{R}$.

Lemma 2 ([1, Lemma 3.2]). Let $\alpha>0$. Then

$$
\alpha|x+y|^{\frac{1+\alpha}{\alpha}} \geq \alpha|y|^{\frac{1+\alpha}{\alpha}}+(1+\alpha) x|y|^{\frac{1}{\alpha}} \operatorname{sgn} y \quad \text { for } x, y \in \mathbb{R} .
$$

Lemma 3. Let $\liminf _{t \rightarrow+\infty} c_{\alpha}(t ; \lambda)>-\infty$ and $(u, v)$ is a solution of system (1.1) satisfying

$$
u(t) \neq 0 \quad \text { for } t \geq t_{u}
$$

with $t_{u}>0$. Then

$$
\int_{t_{u}}^{+\infty} g(s) f^{\lambda-1-\alpha}(s) h(s) d s<+\infty
$$

where

$$
h(t):=\alpha\left|f^{\alpha}(t) \rho(t)-\gamma\right|^{\frac{1+\alpha}{\alpha}}+(1+\alpha) f^{\alpha}(t) \rho(t) \gamma^{\frac{1}{\alpha}}-\alpha \gamma^{\frac{1+\alpha}{\alpha}} \quad \text { for } t \geq t_{u}
$$

the number $\gamma$ is defined by (2.12) and

$$
\rho(t):=\frac{v(t)}{|u(t)|^{\alpha} \operatorname{sgn} u(t)}+\frac{1}{f^{\alpha}(t)}\left(\frac{\lambda}{1+\alpha}\right)^{\alpha} \text { for } t \geq t_{u} .
$$

Proof. Let notice that acording to Lemma 2, we have

$$
h(t) \geq 0 \quad \text { for } t \geq t_{u} .
$$

From the proof [1, Corollary 2.11] one can see that

$$
\liminf _{t \rightarrow+\infty} f^{n(\lambda-\alpha)}(t) \int_{0}^{t}\left[\frac{1}{f^{(\lambda-\alpha)}(t)}-\frac{1}{f^{(\lambda-\alpha)}(s)}\right]^{n} f^{\lambda}(s) p(s) d s>-\infty,
$$

where $n \in \mathbb{N}, n>\max \{1, \alpha\}$. Therefore from [1, Lemma 4.1] we obtain (3.2).

Proof of Theorem 2. Assume on the contrary that system (1.1) is not oscillatory. Then system (1.1) has non-oscillatory solution $(u, v)$, i.e. there exists $t_{u}>0$ such that (3.1) holds. Now we can define the function $\rho$ by (3.4) and from (1.1) we derive that

$$
\rho^{\prime}(t)=-p(t)-\alpha g(t)\left|\rho(t)-\frac{\gamma}{f^{\alpha}(t)}\right|^{\frac{1+\alpha}{\alpha}}+\alpha \gamma \frac{g(t)}{f^{1+\alpha}(t)} \quad \text { for a. e. } t \geq t_{u} .
$$


where the number $\gamma$ is defined by (2.12). Multiplaying the last equality by $f^{\lambda}(t)$ and integrating it from $t_{u}$ to $t$, we obtain

$$
\begin{aligned}
\int_{t_{u}}^{t} f^{\lambda}(s) \rho^{\prime}(s) d s= & -\alpha \int_{t_{u}}^{t} g(s) f^{\lambda-1-\alpha}(s)\left|\rho(s) f^{\alpha}(s)-\gamma\right|^{\frac{1+\alpha}{\alpha}} d s \\
& +\alpha \gamma \int_{t_{u}}^{t} g(s) f^{\lambda-1-\alpha}(s) d s-\int_{t_{u}}^{t} f^{\lambda}(s) p(s) d s \quad \text { for } t \geq t_{u} .
\end{aligned}
$$

Integrating the left-hand side of the last equality by parts, we get

$$
\begin{aligned}
f^{\lambda}(t) \rho(t)= & \left(\alpha \gamma-\alpha \gamma^{\frac{1+\alpha}{\alpha}}\right) \int_{t_{u}}^{t} g(s) f^{\lambda-1-\alpha}(s) d s-\int_{t_{u}}^{t} f^{\lambda}(s) p(s) d s \\
& +f^{\lambda}\left(t_{u}\right) \rho\left(t_{u}\right)-\int_{t_{u}}^{t} g(s) f^{\lambda-1-\alpha}(s) h(s) d s \quad \text { for } t \geq t_{u},
\end{aligned}
$$

where the function $h$ is defined in (3.3). Thus,

$$
\begin{aligned}
f^{\lambda}(t) \rho(t)= & \delta\left(t_{u}\right)-\int_{0}^{t} f^{\lambda}(s) p(s) d s-\int_{t_{u}}^{t} g(s) f^{\lambda-1-\alpha}(s) h(s) d s \\
& -\frac{\alpha\left(\gamma-\gamma^{\frac{1+\alpha}{\alpha}}\right)}{\lambda-\alpha} f^{\lambda-\alpha}(t) \quad \text { for } t \geq t_{u},
\end{aligned}
$$

where

$$
\delta\left(t_{u}\right):=f^{\lambda}\left(t_{u}\right) \rho\left(t_{u}\right)+\int_{0}^{t_{u}} f^{\lambda}(s) p(s) d s+\frac{\alpha\left(\gamma-\gamma^{\frac{1+\alpha}{\alpha}}\right)}{\lambda-\alpha} f^{\lambda-\alpha}\left(t_{u}\right) .
$$

On the other hand one can verify (see Lemma 3 and the proof of [1, Lemma 4.2]) that the finite limit of function $c_{\alpha}(\cdot ; \lambda)$ is

$$
\begin{aligned}
c_{\alpha}^{*}(\lambda)= & f^{\lambda}\left(t_{u}\right) \rho\left(t_{u}\right)+\int_{0}^{t_{u}} f^{\lambda}(s) p(s) d s+\frac{\alpha\left(\gamma-\gamma^{\frac{1+\alpha}{\alpha}}\right)}{\lambda-\alpha} f^{\lambda-\alpha}\left(t_{u}\right) \\
& -\int_{t_{u}}^{+\infty} g(s) f^{\lambda-1-\alpha}(s) h(s) d s .
\end{aligned}
$$

Hence, by virtue of relation (3.2), it follows from (3.7) that

$$
\begin{aligned}
f^{\lambda}(t) \rho(t)= & c_{\alpha}^{*}(\lambda)-\int_{0}^{t} f^{\lambda}(s) p(s) d s+\int_{t}^{+\infty} g(s) f^{\lambda-1-\alpha}(s) h(s) d s \\
& -\frac{\alpha\left(\gamma-\gamma^{\frac{1+\alpha}{\alpha}}\right)}{\lambda-\alpha} f^{\lambda-\alpha}(t) \quad \text { for } t \geq t_{u} .
\end{aligned}
$$


Multiplaying of the last equality by $g(t) f^{\alpha-1-\lambda}(t)$ and integrating it from $t_{u}$ to $t$, we obtain

$$
\begin{aligned}
\int_{t_{u}}^{t} g(s) f^{\alpha-1}(s) \rho(s) d s=-\int_{t_{u}}^{t} \frac{g(s)}{f^{1+\lambda-\alpha}(s)}\left(\int_{0}^{s} f^{\lambda}(\xi) p(\xi) d \xi\right) d s \\
+c_{\alpha}^{*}(\lambda) \int_{t_{u}}^{t} \frac{g(s)}{f^{1+\lambda-\alpha}(s)} d s-\frac{\alpha}{\lambda-\alpha}\left(\gamma-\gamma^{\frac{1+\alpha}{\alpha}}\right) \int_{t_{u}}^{t} \frac{g(s)}{f(s)} d s \\
+\int_{t_{u}}^{t} \frac{g(s)}{f^{1+\lambda-\alpha(s)}}\left(\int_{s}^{+\infty} g(\xi) f^{\lambda-1-\alpha}(\xi) h(\xi) d \xi\right) d s \quad \text { for } t \geq t_{u}
\end{aligned}
$$

One can see

$$
\begin{aligned}
\int_{t_{u}}^{t} & \frac{g(s)}{f^{1+\lambda-\alpha}(s)}\left(\int_{s}^{+\infty} g(\xi) f^{\lambda-1-\alpha}(\xi) h(\xi) d \xi\right) d s \\
\quad= & \frac{1}{(\lambda-\alpha) f^{\lambda-\alpha}(t)} \int_{t}^{+\infty} g(s) f^{\lambda-1-\alpha}(s) h(s) d s+\frac{1}{\lambda-\alpha} \int_{t_{u}}^{t} \frac{g(s)}{f(s)} h(s) d s \\
& -\frac{1}{(\lambda-\alpha) f^{\lambda-\alpha}\left(t_{u}\right)} \int_{t_{u}}^{+\infty} g(s) f^{\lambda-1-\alpha}(s) h(s) d s \quad \text { for } t \geq t_{u} .
\end{aligned}
$$

By virtue of the latter equality, (3.10) yields that

$$
\begin{aligned}
\int_{t_{u}}^{t} g(s) & f^{\alpha-1}(s) \rho(s) d s=\frac{c_{\alpha}^{*}(\lambda)}{\lambda-\alpha}\left[\frac{1}{f^{\lambda-\alpha}(t)}-\frac{1}{f^{\lambda-\alpha}\left(t_{u}\right)}\right]-\frac{1}{(\lambda-\alpha) f^{\lambda-\alpha}(t)} c_{\alpha}(t ; \lambda) \\
& +\frac{1}{(\lambda-\alpha) f^{\lambda-\alpha}\left(t_{u}\right)} c_{\alpha}\left(t_{u} ; \lambda\right)+\frac{1}{(\lambda-\alpha) f^{\lambda-\alpha}(t)} \int_{t}^{+\infty} g(s) f^{\lambda-1-\alpha}(s) h(s) d s \\
& -\frac{1}{(\lambda-\alpha) f^{\lambda-\alpha}\left(t_{u}\right)} \int_{t_{u}}^{+\infty} g(s) f^{\lambda-1-\alpha}(s) h(s) d s+\frac{1}{\lambda-\alpha} \int_{t_{u}}^{t} \frac{g(s)}{f(s)} h(s) d s \\
& -\frac{\alpha}{\lambda-\alpha}\left(\gamma-\gamma^{\frac{1+\alpha}{\alpha}}\right) \int_{t_{u}}^{t} \frac{g(s)}{f(s)} d s \quad \text { for } t \geq t_{u}
\end{aligned}
$$


Hence,

$$
\begin{aligned}
\frac{1}{f^{\lambda-\alpha}(t)}\left(c_{\alpha}^{*}(\lambda)-c_{\alpha}(t ; \lambda)\right) \\
=\int_{t_{u}}^{t} \frac{g(s)}{f(s)}\left[(\lambda-\alpha) f^{\alpha}(s) \rho(s)-h(s)+\alpha\left(\gamma-\gamma^{\frac{1+\alpha}{\alpha}}\right)\right] d s \\
\quad+\frac{1}{f^{\lambda-\alpha}\left(t_{u}\right)}\left[c_{\alpha}^{*}(\lambda)-c_{\alpha}\left(t_{u} ; \lambda\right)+\int_{t_{u}}^{+\infty} g(s) f^{\lambda-1-\alpha}(s) h(s) d s\right] \\
\quad-\frac{1}{f^{\lambda-\alpha}(t)} \int_{t}^{+\infty} g(s) f^{\lambda-1-\alpha}(s) h(s) d s \quad \text { for } t \geq t_{u} .
\end{aligned}
$$

On the other hand, (2.12), (3.3), and Lemma 1 with $\omega:=\alpha$ yield the estimate

$$
\begin{aligned}
& (\lambda-\alpha) f^{\alpha}(s) \rho(s)-h(s)+\alpha\left(\gamma-\gamma^{\frac{1+\alpha}{\alpha}}\right) \\
& \quad=-\alpha\left(f^{\alpha}(s) \rho(s)-\gamma\right)-\alpha\left|f^{\alpha}(s) \rho(s)-\gamma\right|^{\frac{1+\alpha}{\alpha}} \\
& \quad \leq\left(\frac{\alpha}{1+\alpha}\right)^{1+\alpha} \quad \text { for } \quad s \geq t_{u} .
\end{aligned}
$$

Moreover, in view of (1.2), (1.8), and (3.5), it is clear that

$$
\frac{1}{f^{\lambda-\alpha}(t)} \int_{t}^{+\infty} g(s) f^{\lambda-1-\alpha}(s) h(s) d s \geq 0 \quad \text { for } t \geq t_{u} .
$$

Therefore, according to the last inequality and (3.12), it follows from (3.11) that

$$
\begin{gathered}
\frac{1}{f^{\lambda-\alpha}(t)}\left[c_{\alpha}^{*}(\lambda)-c_{\alpha}(t ; \lambda)\right] \leq-\left(\frac{\alpha}{1+\alpha}\right)^{1+\alpha} \ln \frac{f(t)}{f\left(t_{u}\right)} \\
+\frac{1}{f^{\lambda-\alpha}\left(t_{u}\right)}\left[c_{\alpha}^{*}(\lambda)-c_{\alpha}\left(t_{u} ; \lambda\right)+\int_{t_{u}}^{+\infty} g(s) f^{\lambda-1-\alpha}(s) h(s) d s\right] \quad \text { for } t \geq t_{u} .
\end{gathered}
$$

Consequently, in view of (1.7), we get

$$
\limsup _{t \rightarrow+\infty} \frac{-1}{f^{\lambda-\alpha}(t) \ln f(t)}\left[c_{\alpha}^{*}(\lambda)-c_{\alpha}(t ; \lambda)\right] \leq\left(\frac{\alpha}{1+\alpha}\right)^{1+\alpha},
$$

which contradicts (2.1). 
Proof of Corollary 1. It is clear that

$$
\begin{aligned}
c_{\alpha}(t ; \lambda) & =(\lambda-\alpha) f^{\lambda-\alpha}(t) \int_{0}^{t} \frac{g(s)}{f^{1+\lambda-\alpha}(s)}\left(\int_{0}^{s} f^{\lambda}(\xi) p(\xi) d \xi\right) d s \\
& =\int_{0}^{t} f^{\lambda}(s) p(s) d s-f^{\lambda-\alpha}(t) \int_{0}^{t} f^{\alpha}(s) p(s) d s .
\end{aligned}
$$

Hence, by virtue of the definition (2.2), we have

$$
\frac{-1}{f^{\lambda-\alpha}(t) \ln f(t)}\left[c_{\alpha}^{*}(\lambda)-c_{\alpha}(t ; \lambda)\right]=\frac{-Q(t ; \alpha, \lambda)}{\ln f(t)}-\frac{1}{\ln f(t)} \int_{0}^{t} f^{\alpha}(s) p(s) d s
$$

for $t \geq 0$. Now, the last equality, (1.7), (2.5), and the assumption $Q_{*}(\alpha, \lambda)>-\infty$ guarantee the validity of condition (2.1) and thus, the assertion of the corollary follows from Theorem 2.

Proof of Corollary 2. It is not difficult to verify that

$$
\begin{aligned}
& \frac{1}{f^{\lambda-\alpha}(t) \ln f(t)}\left(c_{\alpha}^{*}(\lambda)-c_{\alpha}(t ; \lambda)\right)= \\
& \frac{\lambda-\alpha}{\ln f(t)} \int_{0}^{t} \frac{g(s)}{f(s)} Q(s ; \alpha, \lambda) d s+\frac{c_{\alpha}^{*}(\lambda)}{f^{\lambda-\alpha}(0) \ln f(t)} \quad \text { for } t \geq 0
\end{aligned}
$$

and

$$
\begin{aligned}
& Q(t ; \alpha, \lambda)+H(t ; \alpha, \mu)= \\
& \quad(\lambda-\mu) f^{\alpha-\mu}(t) \int_{0}^{t} g(s) f^{\mu-\alpha-1}(s) Q(s ; \alpha, \lambda) d s+\frac{c_{\alpha}^{*}(\lambda)}{f^{\lambda-\mu}(0)} f^{\alpha-\mu}(t)
\end{aligned}
$$

for $t \geq 0$, where functions $Q(\cdot ; \alpha, \lambda)$ and $H(\cdot ; \alpha, \mu)$ are defined by (2.2) and (2.3). Moreover, it is easy to show that

$$
\begin{aligned}
& \int_{0}^{t} \frac{g(s)}{f(s)} Q(s ; \alpha, \lambda) d s=f^{\alpha-\mu}(t) \int_{0}^{t} g(s) f^{\mu-\alpha-1}(s) Q(s ; \alpha, \lambda) d s \\
& +(\alpha-\mu) \int_{0}^{t} g(s) f^{\alpha-\mu-1}(s)\left(\int_{0}^{s} g(\xi) f^{\mu-\alpha-1}(\xi) Q(\xi ; \alpha, \lambda) d \xi\right) d s
\end{aligned}
$$


for $t \geq 0$. On the other hand, in view of (2.6), relation (3.14) yields

$$
\liminf _{t \rightarrow+\infty} f^{\alpha-\mu}(t) \int_{0}^{t} g(s) f^{\mu-\alpha-1}(s) Q(s ; \alpha, \lambda) d s>\frac{1}{(\lambda-\alpha)(\alpha-\mu)}\left(\frac{\alpha}{\alpha+1}\right)^{\alpha+1} \text {. }
$$

Consequently, in view of (1.7), it follows from (3.15) that

$$
\liminf _{t \rightarrow+\infty} \frac{-1}{\ln f(t)} \int_{0}^{t} \frac{g(s)}{f(s)} Q(s ; \alpha, \lambda) d s>\frac{1}{\lambda-\alpha}\left(\frac{\alpha}{\alpha+1}\right)^{\alpha+1} .
$$

The last inequality, by virtue of (1.7) and (3.13), yields the validity of condition (2.1). Therefore, the assertion of the corollary follows from Theorem 2.

Proof of Corollary 3. First let assumption (2.7) hold. Then it follows from (3.13) that condition (2.1) is satisfied. Therefore, by virtue of Theorem 2 system (1.1) is oscillatory.

Now suppose that assumption (2.8) is fulfilled. It is not difficult to verify that

$$
\int_{0}^{t} f^{\alpha}(s) p(s) d s=H(t ; \alpha, \mu)+(\alpha-\mu) \int_{0}^{t} \frac{g(s)}{f(s)} H(s ; \alpha, \mu) d s \quad \text { for } t \geq 0 .
$$

Hence, in view of (2.8), we get

$$
\liminf _{t \rightarrow+\infty} \frac{-1}{\ln f(t)} \int_{0}^{t} f^{\alpha}(s) p(s) d s>\left(\frac{\alpha}{\alpha+1}\right)^{\alpha+1} .
$$

On the other hand, one can see

$$
c_{\alpha}^{\prime}(t ; \lambda)=\frac{(\lambda-\alpha) g(t)}{f^{\alpha-\lambda+1}(t)} \int_{0}^{t} f^{\alpha}(s) p(s) d s \quad \text { for a. e. } t \geq 0,
$$

where function $c_{\alpha}(\cdot ; \lambda)$ is given by (1.9). By integrating the last equality from $\tau$ to $t$, we obtain

$$
c_{\alpha}(\tau ; \lambda)-c_{\alpha}(t ; \lambda)=(\lambda-\alpha) \int_{t}^{\tau} \frac{g(s)}{f^{\alpha-\lambda+1}(s)}\left(\int_{0}^{s} f^{\alpha}(\xi) p(\xi) d \xi\right) d s \quad \tau \geq t \geq 0
$$

and therefore, by virtue of assumption (1.10) and condition (3.16), we have

$$
\begin{aligned}
& c_{\alpha}^{*}(\lambda)-c_{\alpha}(t ; \lambda)= \\
& \quad(\lambda-\alpha) \int_{t}^{+\infty} \frac{g(s) \ln f(s)}{f^{\alpha-\lambda+1}(s)}\left(\frac{1}{\ln f(s)} \int_{0}^{s} f^{\alpha}(\xi) p(\xi) d \xi\right) d s \quad \text { for } t \geq 0 .
\end{aligned}
$$

In view of (1.7) and (3.16), there exist $\varepsilon>0$ and $t_{\varepsilon}>0$ such that

$$
f(t)<1, \quad \frac{-1}{\ln f(t)} \int_{0}^{t} f^{\alpha}(s) p(s) d s \geq\left(\frac{\alpha}{\alpha+1}\right)^{\alpha+1}+\varepsilon \text { for } t \geq t_{\varepsilon} .
$$


Hence, (3.17) yields that

$$
c_{\alpha}^{*}(\lambda)-c_{\alpha}(t ; \lambda) \geq-(\lambda-\alpha)\left(\left(\frac{\alpha}{\alpha+1}\right)^{\alpha+1}+\varepsilon\right) \int_{t}^{+\infty} \frac{g(s) \ln f(s)}{f^{\alpha-\lambda+1}(s)} \quad \text { for } \quad t \geq t_{\varepsilon} .
$$

By virtue of $\varepsilon>0$ and (1.7), from the last relation we derive inequality (2.1). Consequently, the assertion of the corollary follows from Theorem 2 .

Proof of Theorem 3. Assume on the contrary that system (1.1) is not oscillatory, i.e., there exists a solution $(u, v)$ of system (1.1) satisfying relation (3.1) with $t_{u}>0$. Analogously to the proof of Theorem 2 one can show that equality (3.6) and (3.9) hold, where the number $\gamma$, and the functions $h, \rho$ are given by (2.12), (3.3), and (3.4).

Multiplaying of (3.6) by $f^{\mu}(t)$ and integrating it from $t_{u}$ to $t$, we get

$$
\begin{aligned}
\int_{t_{u}}^{t} f^{\mu}(s) \rho^{\prime}(s) d s= & -\int_{t_{u}}^{t} f^{\mu}(s) p(s) d s-\alpha \int_{t_{u}}^{t} g(s) f^{\mu-\alpha-1}(s)\left|\rho(s) f^{\alpha}(s)-\gamma\right|^{\frac{1+\alpha}{\alpha}} d s \\
& +\alpha \gamma \int_{t_{u}}^{t} g(s) f^{\mu-\alpha-1}(s) d s \quad \text { for } t \geq t_{u} .
\end{aligned}
$$

Now we integrate the left-hand side of the last equality by parts and we obtain

$$
\begin{aligned}
& f^{\alpha}(t) \rho(t)= \\
& \quad+f^{\alpha-\mu}(t) \int_{t_{u}}^{t} g(s) f^{\mu-\alpha-1}(s)\left[-\mu f^{\alpha}(s) \rho(s)-\alpha\left|\rho(s) f^{\alpha}(s)-\gamma\right|^{\frac{1+\alpha}{\alpha}}\right] d s \\
& +\delta\left(t_{u}\right) f^{\alpha-\mu}(t)-H(t ; \alpha, \mu)+\frac{\alpha \gamma}{\alpha-\mu} \quad \text { for } t \geq t_{u},
\end{aligned}
$$

where the function $H(\cdot ; \alpha, \mu)$ is defined by $(2.3)$ and

$$
\delta\left(t_{u}\right):=f^{\mu}\left(t_{u}\right) \rho\left(t_{u}\right)+\int_{0}^{t_{u}} f^{\mu}(s) p(s) d s-\frac{\alpha \gamma}{(\alpha-\mu)} \frac{1}{f^{\alpha-\mu}\left(t_{u}\right)} .
$$

On the other hand, multiplaying of (3.9) by $f^{\alpha-\lambda}(t)$, we get

$$
\begin{aligned}
f^{\alpha}(t) \rho(t)= & Q(t ; \alpha, \lambda)+\frac{1}{f^{\lambda-\alpha}(t)} \int_{t}^{+\infty} g(s) f^{\lambda-1-\alpha}(s) h(s) d s \\
& -\frac{\alpha\left(\gamma-\gamma^{\frac{1+\alpha}{\alpha}}\right)}{\lambda-\alpha} \text { for } t \geq t_{u},
\end{aligned}
$$


where the function $Q(\cdot ; \alpha, \lambda)$ is defined by (2.2). Hence, by virtue of (3.18), one can see

$$
\begin{aligned}
& Q(t ; \alpha, \lambda)+H(t ; \alpha, \mu)=-\frac{1}{f^{\lambda-\alpha}(t)} \int_{t}^{+\infty} g(s) f^{\lambda-1-\alpha}(s) h(s) d s \\
& +f^{\alpha-\mu}(t) \int_{t_{u}}^{t} g(s) f^{\mu-\alpha-1}(s)\left[-\mu f^{\alpha}(s) \rho(s)-\alpha\left|\rho(s) f^{\alpha}(s)-\gamma\right|^{\frac{1+\alpha}{\alpha}}\right] d s \\
& +\frac{\alpha}{\lambda-\alpha}\left(\gamma-\gamma^{\frac{1+\alpha}{\alpha}}\right)+\frac{\alpha \gamma}{\alpha-\mu}+\delta\left(t_{u}\right) f^{\alpha-\mu}(t) \quad \text { for } t \geq t_{u} .
\end{aligned}
$$

Moreover, it follows Lemma 1 with $\omega:=\mu \geq 0$ that

$$
-\mu\left(f^{\alpha}(t) \rho(t)-\gamma\right)-\alpha\left|\rho(t) f^{\alpha}(t)-\gamma\right|^{\frac{1+\alpha}{\alpha}} \leq\left(\frac{\mu}{1+\alpha}\right)^{1+\alpha} \quad \text { for } t \geq t_{u} .
$$

Therefore, using (2.12), (3.5), and (3.21) in relation (3.20) yields

$$
\begin{aligned}
Q(t ; \alpha, \lambda)+H(t ; \alpha, \mu) \leq & \frac{1}{\lambda-\alpha}\left(\frac{\lambda}{1+\alpha}\right)^{1+\alpha}+\frac{1}{\alpha-\mu}\left(\frac{\mu}{1+\alpha}\right)^{1+\alpha} \\
& +\delta_{1}\left(t_{u}\right) f^{\alpha-\mu}(t) \quad \text { for } t \geq t_{u}
\end{aligned}
$$

where

$$
\delta_{1}\left(t_{u}\right):=\delta\left(t_{u}\right)+\left[\mu \gamma-\left(\frac{\mu}{1+\alpha}\right)^{1+\alpha}\right] \frac{1}{(\alpha-\mu) f^{\alpha-\mu}\left(t_{u}\right)} .
$$

Consequently, by virtue of (1.7) relation (3.22) contradicts with assumption (2.9).

Proof of Theorem 4. Assume on the contrary that system (1.1) is not oscillatory. Then there exists a solution $(u, v)$ of system (1.1) satisfying relation (3.1) with $t_{u}>0$. Analogously to the proof of Theorem 3 one can show that relation (3.18) holds, where the numbers $\gamma, \delta\left(t_{u}\right)$ and the functions $H(\cdot ; \alpha, \mu), \rho$ are given by (2.12), (3.19), (2.3), and (3.4). Moreover using inequality (3.21) in (3.18) yields

$$
f^{\alpha}(t) \rho(t) \leq \delta_{1}\left(t_{u}\right) f^{\alpha-\mu}(t)-H(t ; \alpha, \mu)+\frac{1}{\alpha-\mu}\left(\frac{\mu}{1+\alpha}\right)^{1+\alpha}+\gamma \quad \text { for } t \geq t_{u},
$$

where $\delta_{1}\left(t_{u}\right)$ is given by (3.23).

Now we show that the estimate

$$
\liminf _{t \rightarrow+\infty} f^{\alpha}(t) \rho(t) \geq A(\alpha, \lambda)
$$

holds, where $A(\alpha, \lambda)$ denotes the smallest root of equation (2.13). Similarly, as in the proof of Theorem 2 we can derive that equality (3.8) holds. Multiplying (3.8) by 
$f^{\alpha-\lambda}(t)$, we obtain

$$
\begin{aligned}
f^{\alpha}(t) \rho(t)= & Q(t ; \alpha, \lambda)+\frac{1}{f^{\lambda-\alpha}(t)} \int_{t}^{+\infty} g(s) f^{\lambda-1-\alpha}(s) h(s) d s \\
& -\frac{\alpha\left(\gamma-\gamma^{\frac{1+\alpha}{\alpha}}\right)}{\lambda-\alpha} \quad \text { for } t \geq t_{u} .
\end{aligned}
$$

Let denote

$$
m:=\liminf _{t \rightarrow+\infty} f^{\alpha}(t) \rho(t) .
$$

Observe that, if $m=+\infty$, then (3.25) is fulfilled. Therefore, we assume

$$
m<+\infty \text {. }
$$

In view of (2.10), (3.5), and (3.27), it follows from relation (3.26)

$$
m \geq Q_{*}(\alpha, \lambda)-\frac{\alpha}{\lambda-\alpha}\left(\gamma-\gamma^{\frac{1+\alpha}{\alpha}}\right) \geq 0,
$$

where $Q_{*}(\alpha, \lambda)$ is given by (2.4).

First suppose that $Q_{*}(\alpha, \lambda)=\frac{\alpha}{\lambda-\alpha}\left(\gamma-\gamma^{\frac{1+\alpha}{\alpha}}\right)$, then it is clear that 0 is a root of equation (2.13). Moreover, in view of Lemma 2 and the assumption $\lambda>\alpha$, one can derive that the function $x \mapsto \alpha|x-\gamma|^{\frac{1+\alpha}{\alpha}}+\alpha x-\alpha \gamma^{\frac{1+\alpha}{\alpha}}$ is positive on $]-\infty, 0[$. Consequently, by virtue of notation (3.27) and relation (3.28), desired estimate (3.25) holds.

Now suppose that $Q_{*}(\alpha, \lambda)>\frac{\alpha}{\lambda-\alpha}\left(\gamma-\gamma^{\frac{1+\alpha}{\alpha}}\right)$. Let $\varepsilon \in] 0, Q_{*}(\alpha, \lambda)-\frac{\alpha}{\lambda-\alpha}\left(\gamma-\gamma^{\frac{1+\alpha}{\alpha}}\right)[$ be arbitrary. According to (3.28), it is clear that

$$
m>\varepsilon .
$$

In view of (2.4) and (3.27), there exists $t_{\varepsilon} \geq t_{u}$ such that

$$
f^{\alpha}(t) \rho(t) \geq m-\varepsilon \quad \text { and } \quad Q(t ; \alpha, \lambda) \geq Q_{*}(\alpha, \lambda)-\varepsilon \quad \text { for } t \geq t_{\varepsilon} .
$$

Then (3.26) yields that

$$
\begin{aligned}
f^{\alpha}(t) \rho(t) \geq & Q_{*}(\alpha, \lambda)-\varepsilon+\frac{1}{f^{\lambda-\alpha}(t)} \int_{t}^{+\infty} g(s) f^{\lambda-1-\alpha}(s) h(s) d s \\
& -\frac{\alpha\left(\gamma-\gamma^{\frac{1+\alpha}{\alpha}}\right)}{\lambda-\alpha} \text { for } t \geq t_{\varepsilon} .
\end{aligned}
$$

On the other hand, one can see that the function $x \mapsto \alpha|x-\gamma|^{\frac{1+\alpha}{\alpha}}+(1+\alpha) x \gamma^{\frac{1}{\alpha}}-$

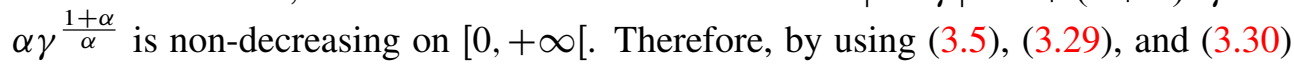
in (3.31), we obtain that

$$
f^{\alpha}(t) \rho(t) \geq Q_{*}(\alpha, \lambda)-\varepsilon+\frac{\alpha|(m-\varepsilon)-\gamma|^{\frac{1+\alpha}{\alpha}}-\alpha \gamma+\lambda(m-\varepsilon)}{\lambda-\alpha} \quad \text { for } t \geq t_{\varepsilon},
$$


which implies

$$
m \geq Q_{*}(\alpha, \lambda)-\varepsilon+\frac{\alpha|(m-\varepsilon)-\gamma|^{\frac{1+\alpha}{\alpha}}-\alpha \gamma+\lambda(m-\varepsilon)}{\lambda-\alpha} .
$$

Since $\varepsilon$ was arbitrary, the latter relation leads to the inequality

$$
\alpha|m-\gamma|^{\frac{1+\alpha}{\alpha}}+\alpha m+Q_{*}(\alpha, \lambda)(\lambda-\alpha)-\alpha \gamma \leq 0 .
$$

One can show that the function $y: x \mapsto \alpha|x-\gamma|^{\frac{1+\alpha}{\alpha}}+\alpha x+Q_{*}(\alpha, \lambda)(\lambda-\alpha)-\alpha \gamma$ is decreasing on $\left.]-\infty, \gamma-\left(\frac{\alpha}{1+\alpha}\right)^{\alpha}\right]$ and increasing on $\left[\gamma-\left(\frac{\alpha}{1+\alpha}\right)^{\alpha},+\infty[\right.$. Moreover, in view of assumption (2.10), the function $y$ is non-positive at the point $\gamma-\left(\frac{\alpha}{1+\alpha}\right)^{\alpha}$, which together with (3.27), and (3.32) yields desired estimate (3.25).

Let now $\widetilde{\varepsilon}>0$ be arbitrary. In view of (3.25) there exists $t_{\varepsilon} \geq t_{u}$ such that

$$
f^{\alpha}(t) \rho(t) \geq A(\alpha, \lambda)-\widetilde{\varepsilon} \text { for } t \geq \tau_{\mathcal{\varepsilon}},
$$

Hence, it follows from (3.24) that

$$
H(t ; \alpha, \mu) \leq \delta_{1}\left(t_{u}\right) f^{\alpha-\mu}(t)-A(\alpha, \lambda)+\widetilde{\varepsilon}+\frac{1}{\alpha-\mu}\left(\frac{\mu}{1+\alpha}\right)^{1+\alpha}+\gamma \quad \text { for } t \geq \tau_{\mathcal{\varepsilon}} .
$$

Since $\widetilde{\varepsilon}$ was arbitrary, in view of (1.7) and (2.4), from the latter inequality we obtain

$$
H^{*}(\alpha, \mu) \leq \frac{1}{\alpha-\mu}\left(\frac{\mu}{1+\alpha}\right)^{1+\alpha}+\gamma-A(\alpha, \lambda, \gamma),
$$

which contradicts assumption (2.11).

Proof of Theorem 5. Assume on the contrary that system (1.1) is not oscillatory, i.e., there exists a solution $(u, v)$ of system (1.1) satisfying relation (3.1) with $t_{u}>0$.

First we show that the estimate

$$
\limsup _{t \rightarrow+\infty}\left(f^{\alpha}(t) \rho(t)-\gamma\right) \leq B(\alpha, \mu),
$$

holds, where the number $\gamma$, the function $\rho$ are defined by (2.12), (3.4), and $B(\alpha, \mu)$ is the greatest root of equation (2.16). Let denote

$$
M:=\limsup _{t \rightarrow+\infty}\left(f^{\alpha}(t) \rho(t)-\gamma\right) .
$$

It is clear that, if $M=-\infty$ then (3.33) holds. Therefore, we suppose that

$$
M>-\infty \text {. }
$$

Analogously to the proof of Theorem 4, we can derive inequality (3.24), where the function $H(t ; \alpha, \mu)$ is defined by (2.3). Then, in view of (1.7) and (3.34), it follows from inequality (3.24) that

$$
M \leq-H_{*}(\alpha, \mu)+\frac{1}{\alpha-\mu}\left(\frac{\mu}{1+\alpha}\right)^{1+\alpha} .
$$


First we assume that $H_{*}(\alpha, \mu)=\left(\frac{\mu}{1+\alpha}\right)^{\alpha} \frac{\alpha(1+\alpha-\mu)}{(\alpha-\mu)(1+\alpha)}$. Then it is not difficult to verify that $-\left(\frac{\mu}{1+\alpha}\right)^{\alpha}$ is a root of the equation (2.16) and the function $x \mapsto \alpha|x|^{\frac{1+\alpha}{\alpha}}+$ $\alpha x+(\alpha-\mu) H_{*}(\alpha, \mu)$ is positive on $]-\left(\frac{\mu}{1+\alpha}\right)^{\alpha},+\infty[$. Therefore, (3.34) and (3.35) yields that (3.33) is satisfied.

Now we suppose

$$
H_{*}(\alpha, \mu)>\left(\frac{\mu}{1+\alpha}\right)^{\alpha} \frac{\alpha(1+\alpha-\mu)}{(\alpha-\mu)(1+\alpha)} .
$$

Hence, it follows from (3.35)

$$
M<-\left(\frac{\mu}{1+\alpha}\right)^{\alpha}
$$

On the other hand, similarly as in the proof of the Theorem 3 we can derive equality (3.18). Let $\varepsilon \in] 0,-\left(\frac{\mu}{1+\alpha}\right)^{\alpha}-M\left[\right.$ be arbitrary and choose $t_{\varepsilon} \geq t_{u}$ such that

$$
f^{\alpha}(t) \rho(t)-\gamma \leq M+\varepsilon, \quad H(t ; \alpha, \mu) \geq H_{*}(\alpha, \mu)-\varepsilon \quad \text { for } t \geq t_{\varepsilon} .
$$

One can see that the function $x \mapsto \mu x+\alpha|x|^{\frac{1+\alpha}{\alpha}}$ is non-increasing on interval ] $\left.\infty,-\left(\frac{\mu}{1+\alpha}\right)^{\alpha}\right]$ and thus, using relations (3.36) and $M+\varepsilon<-\left(\frac{\mu}{1+\alpha}\right)^{\alpha}$ in (3.18), we get

$$
\begin{aligned}
& f^{\alpha}(t) \rho(t) \leq \delta_{2}\left(t_{u}\right) f^{\alpha-\mu}(t)-H_{*}(\alpha, \mu)+\varepsilon+\frac{\alpha \gamma}{\alpha-\mu}-\frac{\mu \gamma}{\alpha-\mu} \\
& \quad+f^{\alpha-\mu}(t) \int_{t_{u}}^{t} g(s) f^{\mu-\alpha-1}(s)\left[-\mu(M+\varepsilon)-\alpha|M+\varepsilon|^{\frac{1+\alpha}{\alpha}}\right] d s \quad \text { for } t \geq t_{\varepsilon},
\end{aligned}
$$

where

$$
\delta_{2}\left(t_{u}\right):=f^{\mu}\left(t_{u}\right) \rho\left(t_{u}\right)+\int_{0}^{t_{u}} f^{\mu}(s) p(s) d s-\gamma \frac{1}{f^{\alpha-\mu}\left(t_{u}\right)} .
$$

Hence,

$$
\begin{aligned}
f^{\alpha}(t) \rho(t)-\gamma \leq & \delta_{3}\left(t_{u}\right) f^{\alpha-\mu}(t)-H_{*}(\alpha, \mu)+\varepsilon \\
& -\frac{\mu(M+\varepsilon)+\alpha|M+\varepsilon|^{\frac{1+\alpha}{\alpha}}}{\alpha-\mu} \text { for } t \geq t_{\varepsilon},
\end{aligned}
$$

where

$$
\delta_{3}\left(t_{u}\right):=\delta_{2}\left(t_{u}\right)+\frac{\mu(M+\varepsilon)+\alpha|M+\varepsilon|^{\frac{1+\alpha}{\alpha}}}{(\alpha-\mu)} \frac{1}{f^{\alpha-\mu}\left(t_{u}\right)},
$$

which, by virtue of the assumption $\alpha>\mu$, condition (1.7) and notation (3.34), yields that

$$
M \leq-H_{*}(\alpha, \mu)+\varepsilon-\frac{\mu(M+\varepsilon)+\alpha|M+\varepsilon|^{\frac{1+\alpha}{\alpha}}}{\alpha-\mu} .
$$


Because of $\varepsilon$ was choosen arbitrary, from the latter inequality follows

$$
\alpha|M|^{\frac{1+\alpha}{\alpha}}+\alpha M+(\alpha-\mu) H_{*}(\alpha, \mu) \leq 0 .
$$

One can easily verify that the function $y: x \mapsto \alpha|x|^{\frac{1+\alpha}{\alpha}}+\alpha x+H_{*}(\alpha, \mu)(\alpha-\mu)$ is decreasing on $\left.]-\infty,-\left(\frac{\alpha}{1+\alpha}\right)^{\alpha}\right]$ and increasing on $\left[-\left(\frac{\alpha}{1+\alpha}\right)^{\alpha},+\infty[\right.$. Moreover, in view of assumption (2.14), the function $y$ is non-positive at the point $-\left(\frac{\alpha}{1+\alpha}\right)^{\alpha}$, which together with (3.34), and (3.37) yields desired estimate (3.33).

Let now $\widetilde{\varepsilon}>0$ be arbitrary. Then, by virtue of (3.33), there exists $\tau_{\varepsilon} \geq t_{u}$ such that

$$
f^{\alpha}(t) \rho(t)-\gamma \leq B(\alpha, \mu)+\widetilde{\varepsilon} \text { for } t \geq \tau_{\mathcal{\varepsilon}}
$$

Observe that, analogously to the proof of Theorem 4 we can derive relation (3.26). Then, in view of the last inequality, (1.2), (1.8) and (3.5), it follows from (3.26)

$$
Q(t ; \alpha, \lambda) \leq B(\alpha, \mu)+\widetilde{\varepsilon}+\gamma+\frac{\alpha}{\lambda-\alpha}\left(\gamma-\gamma^{\frac{1+\alpha}{\alpha}}\right) \text { for } t \geq \tau_{\varepsilon} .
$$

Since $\widetilde{\varepsilon}$ was arbitrary, from the last inequality and (2.4), we obtain

$$
Q^{*}(\alpha, \lambda) \leq B(\alpha, \mu)+\frac{\gamma^{\frac{1+\alpha}{\alpha}}}{\lambda-\alpha},
$$

which is in contradiction with (2.15).

Proof of Theorem 6. Suppose on the contrary that system (1.1) is not oscillatory. Then there exists a solution $(u, v)$ of system (1.1) satisfying relation (3.1) with $t_{u}>0$. Put

$$
\widetilde{m}:=A(\alpha, \lambda), \quad \widetilde{M}:=B(\alpha, \mu),
$$

i.e., $\widetilde{m}$ denotes the smallest root of equation (2.13) and $\widetilde{M}$ is the greatest root of equation (2.16). Analogously to the proofs of Theorems 4 and 5 we can derive that estimates (3.25) and (3.33) hold. Consequently, by virtue of (3.38), we have

$$
\liminf _{t \rightarrow+\infty} f^{\alpha}(t) \rho(t) \geq \widetilde{m}, \quad \limsup _{t \rightarrow+\infty}\left(f^{\alpha}(t) \rho(t)-\gamma\right) \leq \widetilde{M},
$$

where the number $\gamma$ and the function $\rho$ are defined by (2.12) and (3.4).

On the other hand, in view of (2.10), one can show that the function $y: x \mapsto$ $\alpha|x-\gamma|^{\frac{1+\alpha}{\alpha}}+\alpha x+Q_{*}(\alpha, \lambda)(\lambda-\alpha)-\alpha \gamma$ is positive on $]-\infty, 0[$ and there exists $\bar{x} \in$ $[0,+\infty[$ such that $y(\bar{x}) \leq 0$, which yields that $\widetilde{m} \geq 0$. Moreover, by virtue of (2.14), one can easily verify that the function $z: x \mapsto \alpha|x|^{\frac{1+\alpha}{\alpha}}+\alpha x+(\alpha-\mu) H_{*}(\alpha, \mu)$ is positive on $]-\left(\frac{\mu}{1+\alpha}\right)^{\alpha},+\infty\left[\right.$ and there exists $\tilde{x} \leq-\left(\frac{\mu}{1+\alpha}\right)^{\alpha}$ such that $z(\tilde{x}) \leq 0$. Consequently, we have $\widetilde{M} \leq-\left(\frac{\mu}{1+\alpha}\right)^{\alpha}$.

We first assume that $\widetilde{m}>0$ and $\widetilde{M}<-\left(\frac{\mu}{1+\alpha}\right)^{\alpha}$. Let $\left.\varepsilon \in\right] 0, \min \left\{\widetilde{m},-\left(\frac{\mu}{1+\alpha}\right)^{\alpha}-\right.$ $\widetilde{M}\}$ [ be arbitrary. Then, it follows from (3.39) that, there exists $t_{\varepsilon} \geq t_{u}$ such that

$$
f^{\alpha}(t) \rho(t) \geq \widetilde{m}-\varepsilon, \quad f^{\alpha}(t) \rho(t)-\gamma \leq \widetilde{M}+\varepsilon \quad \text { for } t \geq t_{\varepsilon} .
$$


Observe that, the function $x \mapsto \alpha|x-\gamma|^{\frac{1+\alpha}{\alpha}}+(1+\alpha) x \gamma^{\frac{1}{\alpha}}$ is non-decrasing on $[0,+\infty[$ and thus, in view of (3.3) and (3.40), we obtain

$$
\begin{aligned}
& \frac{1}{f^{\lambda-\alpha}(t)} \int_{t}^{+\infty} g(s) f^{\lambda-1-\alpha}(s) h(s) d s \geq \\
& \quad+\frac{\alpha|\widetilde{m}-\varepsilon-\gamma|^{\frac{1+\alpha}{\alpha}}+\lambda(\widetilde{m}-\varepsilon)-\alpha \gamma^{\frac{1+\alpha}{\alpha}}}{\lambda-\alpha} \text { for } t \geq t_{\varepsilon} .
\end{aligned}
$$

Moreover, the function $x \mapsto-\mu x-\alpha|x|^{\frac{1+\alpha}{\alpha}}$ is non-decrasing on $]-\infty,-\left(\frac{\mu}{1+\alpha}\right)^{\alpha}[$. Therefore, by virtue of (3.40), we obtain

$$
\begin{gathered}
f^{\alpha-\mu}(t) \int_{t_{\varepsilon}}^{t} g(s) f^{\mu-\alpha-1}(s)\left[-\mu f^{\alpha}(s) \rho(s)-\alpha\left|\rho(s) f^{\alpha}(s)-\gamma\right|^{\frac{1+\alpha}{\alpha}}\right] d s \\
\leq \frac{-\mu(\widetilde{M}+\varepsilon)-\alpha|\widetilde{M}+\varepsilon|^{\frac{1+\alpha}{\alpha}}-\mu \gamma}{\alpha-\mu} \text { for } t \geq t_{\varepsilon} .
\end{gathered}
$$

On the other hand, analogously to the proof of Theorem 3 we can derive relation (3.20), where the number $\delta\left(t_{u}\right)$ and the function $h$ are defined by (3.19) and (3.3). Now it follows from (3.20), (3.41), and (3.42)

$$
\begin{aligned}
Q(t ; \alpha, \lambda) & +H(t ; \alpha, \mu) \leq \widetilde{M}+\varepsilon+H_{*}(\alpha, \mu)-(\widetilde{m}-\varepsilon)+Q_{*}(\alpha, \lambda)+\gamma \\
& -\frac{\alpha(\widetilde{M}+\varepsilon)+\alpha|\widetilde{M}+\varepsilon|^{\frac{1+\alpha}{\alpha}}+(\alpha-\mu) H_{*}(\alpha, \mu)}{\alpha-\mu} \\
& -\frac{\alpha|\widetilde{m}-\varepsilon-\gamma|^{\frac{1+\alpha}{\alpha}}+\alpha(\widetilde{m}-\varepsilon)+(\lambda-\alpha) Q_{*}(\alpha, \lambda)-\alpha \gamma}{\lambda-\alpha} \\
& +\delta\left(t_{\varepsilon}\right) f^{\alpha-\mu}(t) \quad \text { for } t \geq t_{\varepsilon},
\end{aligned}
$$

where

$$
\delta\left(t_{\varepsilon}\right):=\delta\left(t_{u}\right)+\int_{t_{u}}^{t_{\varepsilon}} g(s) f^{\mu-\alpha-1}(s)\left[-\mu f^{\alpha}(s) \rho(s)-\alpha\left|\rho(s) f^{\alpha}(s)-\gamma\right|^{\frac{1+\alpha}{\alpha}}\right] d s .
$$

Since $\varepsilon$ was arbitrary, in view of (1.7) and (3.38), inequality (3.43) yields that

$$
\begin{aligned}
\limsup _{t \rightarrow+\infty}(Q(t ; \alpha, \lambda)+H(t ; \alpha, \mu)) \leq & B(\alpha, \mu)-A(\alpha, \lambda, \gamma) \\
& +Q_{*}(\alpha, \lambda)+H_{*}(\alpha, \mu)+\gamma
\end{aligned}
$$

which contradicts assumption (2.17).

If $\widetilde{m}=0$ then, in view of (3.5), it is clear that

$$
\frac{1}{f^{\lambda-\alpha}(t)} \int_{t}^{+\infty} g(s) f^{\lambda-1-\alpha}(s) h(s) d s \geq 0=\frac{\alpha|\widetilde{m}-\gamma|^{\frac{1+\alpha}{\alpha}}+\lambda \widetilde{m}-\alpha \gamma^{\frac{1+\alpha}{\alpha}}}{\alpha-\lambda}
$$


for $t \geq t_{u}$. On the other hand, if $\widetilde{M}=-\left(\frac{\mu}{1+\alpha}\right)^{\alpha}$ then, using Lemma 1 with $\omega:=\mu$, one can show that

$$
\begin{aligned}
& f^{\alpha-\mu}(t) \int_{t_{u}}^{t} g(s) f^{\mu-\alpha-1}(s)\left[-\mu f^{\alpha}(s) \rho(s)-\alpha\left|\rho(s) f^{\alpha}(s)-\gamma\right|^{\frac{1+\alpha}{\alpha}}\right] d s \\
& \leq \frac{\left(\frac{\mu}{1+\alpha}\right)^{1+\alpha}-\mu \gamma}{\alpha-\mu}-\frac{f^{\alpha-\mu}(t)}{f^{\alpha-\mu}\left(t_{u}\right)}\left[\frac{\left(\frac{\mu}{1+\alpha}\right)^{1+\alpha}-\mu \gamma}{\alpha-\mu}\right] \\
& =\frac{-\mu \widetilde{M}-\alpha|\widetilde{M}|^{\frac{1+\alpha}{\alpha}}-\mu \gamma}{\alpha-\mu}-\frac{f^{\alpha-\mu}(t)}{f^{\alpha-\mu}\left(t_{u}\right)}\left[\frac{\left(\frac{\mu}{1+\alpha}\right)^{1+\alpha}-\mu \gamma}{\alpha-\mu}\right] \quad \text { for } t \geq t_{u} .
\end{aligned}
$$

Consequently, if $\widetilde{m}=0$ (resp. $\left.\widetilde{M}=-\left(\frac{\mu}{1+\alpha}\right)^{\alpha}\right)$, then we derive the inequality (3.44) from (3.20) similarly as above, but we use (3.45) instead of (3.41) (resp. (3.46) instead of (3.42)).

\section{REFERENCES}

[1] M. Dosoudilová, A. Lomtatidze, and J. Šremr, "Oscillatory properties of solutions to certain twodimensional systems of non-linear ordinary differential equations." Nonlinear Analysis: Theory, Methods Applications, vol. 120, pp. 57-75, 2015, doi: 10.1016/j.na.2015.02.014.

[2] O. Došlý and P. Řehák, Half-linear differential equations. Amsterdam: Elsevir Science B.V., 2005.

[3] N. Kandelaki, A. Lomtatidze, and D. Ugalva, "On oscillation and nonoscillation of a second order half-linear equation." Georgian Math. J., vol. 7, no. 2, pp. 329-346, 2000.

[4] T. Kusano and Y. Naito, "Oscillation and nonoscillation criteria for second order quasilinear differential equations," Acta Math. Hungar., vol. 76, no. 1-2, pp. 81-99, 1997, doi: 10.1007/BF02907054.

[5] T. Kusano and J. Wang, "Oscillation properties of half-linear functional-differential equations of the second order," Hiroshima Math. J., vol. 25, no. 2, pp. 371-385, 1995.

[6] A. Lomtatidze and J. Šremr, "On oscillation and nonoscillation of two-dimensional linear differential systems," Georgian Math. J., vol. 20, no. 3, pp. 573-600, 2013, doi: 10.1515/gmj-2013-0025.

[7] J. D. Mirzov, "On some analogs of Sturm's Kneser's theorems for nonlinear systems," J. Math. Anal. Appl., vol. 53, no. 2, pp. 418-425, 1976, doi: 10.1016/0022-247X(76)90120-7.

[8] J. D. Mirzov, Asymptotic properties of solutions of systems of nonlinear nonautonomous ordinary differential equations. Brno: Masaryk University, Brno, 2004.

[9] P. Rehak, "A Riccati technique for proving oscillation of a half-linear equation." Electron. J. Differ. Equ., vol. 2008, pp. 105-113, 2008.

\section{Author's address}

\section{Zdeněk Opluštil}

Institute of Mathematics, Faculty of Mechanical Engineering, Brno University of Technology, Technická 2, 61669 Brno, Czech Republic 